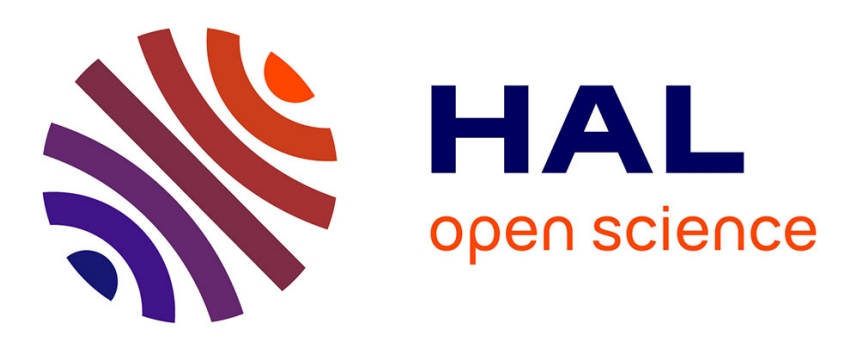

\title{
Adaptive analysis in elastoplasticity based on an enhanced error in constitutive relation
}

\author{
L. Gallimard, J.P. P Pelle
}

\section{To cite this version:}

L. Gallimard, J.P. P Pelle. Adaptive analysis in elastoplasticity based on an enhanced error in constitutive relation. Engineering Computations, 2001, 18 (7), pp.974 - 995. 10.1108/02644400110403993 . hal-01689632

\section{HAL Id: hal-01689632 \\ https://hal.parisnanterre.fr/hal-01689632}

Submitted on 22 Jan 2018

HAL is a multi-disciplinary open access archive for the deposit and dissemination of scientific research documents, whether they are published or not. The documents may come from teaching and research institutions in France or abroad, or from public or private research centers.
L'archive ouverte pluridisciplinaire HAL, est destinée au dépôt et à la diffusion de documents scientifiques de niveau recherche, publiés ou non, émanant des établissements d'enseignement et de recherche français ou étrangers, des laboratoires publics ou privés. 


\title{
Adaptive analysis in elastoplasticity based on an enhanced error in constitutive relation
}

\author{
L. Gallimard and J.P. Pelle \\ LMT Cachan, ENS de CACHAN/CNRS/Université Paris VI, Cachan \\ France
}

Keywords Finite element analysis, Error cause removal, Plasticity

Abstract Many industrial analyses require the resolution of complex nonlinear problems. For such calculations, error-controlled adaptive strategies must be used to improve the quality of the results. In this paper, adaptive strategies for nonlinear calculations in plasticity based on an enhanced error on the constitutive relation are presented. We focus on the adaptivity of the mesh and of the time discretization.

\section{Introduction}

In elastoplasticity, the quality of the finite element solution depends on the parameters describing the discretizations used: the mesh elements' sizes, the order of the elements, the size of the time steps, but also the parameters of the iterative techniques used to solve the nonlinear problems (particularly the stopping criteria for the iterations). In elastoplasticity, low-degree elements (first- or second-degree) are generally used. Moreover, when the stopping criteria are sufficiently precise, the error introduced by the iterative techniques is negligible in comparison with the errors due to the mesh and the time discretization (Ladevèze et al., 1999). For these reasons, improving the quality of the finite element analysis requires, in most cases, simultaneous adaptations of the mesh and of the time discretization (Gallimard et al., 1996). A classical approach is to perform an initial finite element analysis and to calculate a posteriori error estimators and error indicators on this first analysis. These errors are then used to determine new computation parameters in order to improve the quality of the calculation. The efficiency of the adaptive procedure depends greatly on the accuracy of the information given by the error estimator, both on the global level and on the local level. Three main approaches to the development of estimators can be found in the literature: the estimators introduced by Babuška and Rheinboldt (1978), which use the equilibrium residuals to calculate the errors (Babuška and Rheinboldt, 1982; Johnson and Hansbo, 1992; Huerta et al., 1998; Rannacher and Stuttmeier, 1999); the estimators introduced by Zienkiewicz (Zienkiewicz and Zhu, 1987), which consist of comparing the finite element solution with a smoothed solution (Zienkiewicz and Zhu, 1987; Coupez et al., 1998); the estimators introduced by Ladevèze (Ladevèze and Pelle, 1983), which are 
based on the concept of error in the constitutive relation (Ladevèze et al., 1986; Gallimard et al., 1996; 1997, 2000; Ladevèze and Moës, 1998; Ladevèze et al., 1999). The error measures in the constitutive relation satisfies the following property: if the error measure is equal to zero then the approximate solution of the problem is equal to the exact one. That means that this error measure takes into account all discretization errors over the whole time interval.

The purpose of the paper is, for finite element analysis in elastoplasticity, to compare the efficiency of adaptive techniques based on different versions of Drucker's (1964) error in the constitutive relation. Three versions are compared:

(1) A version, called standard, introduced in Ladevèze et al. (1986) and developed in Gallimard et al. (1996), which uses a rigorous quasi-explicit construction of the admissible fields.

(2) A version, called " $p+k$ ", which uses an approximate numerical construction of the admissible fields. This version is an extension to elastoplasticity of techniques used in heat transfer by Strouboulis in Strouboulis and Haque (1992) and in linear elasticity in Coorevits et al. (1999).

(3) An enhanced version developed in Gallimard et al. (2000), where we introduce a minimization of the error on a set of admissible stress fields which leads to an enhanced admissible stress field which is nearer the exact stress field that the admissible field obtained by the standard and the " $p+k$ " version. This version leads to better global and local effectivity indexes than the previous two.

In the first section, we briefly recall the formulation of the elastoplasticity problem which serves as reference. Then, the formulation of the associated discretized problem is given. Drucker's error on the constitutive relation is recalled, along with the principle of its implementation: construction of balanced force densities and construction of admissible stresses. The enhanced version and its different variants are presented and compared. The last section is devoted to the adaptation of the finite element analysis. To separate, in the global error, the contribution due to the spatial discretization from the contribution due to the time discretization, error indicators are proposed. The examples of adaptation presented confirm the effectiveness of the enhanced error estimator introduced in Gallimard et al. (2000).

\section{Problem to be solved}

Let us consider a structure $\Omega$, with boundary $\partial \Omega$. Over the interval $[0, T]$ the structure is subjected to:

- a prescribed displacement on a part $\partial_{1} \Omega$ of the boundary: $u_{d}(M, t)$;

- a force density given by $\partial_{2} \Omega=\partial \Omega-\partial_{1} \Omega: F_{d}(M, t)$;

- a density of body forces in $\Omega: f_{d}(M, t)$. 
The problem which describes the evolution of the structure under the assumption of small perturbations is:

Find $u(M, t)$ and $\sigma(M, t)$ defined on $\Omega \times[0, T]$ such that:

- kinematic constraints and initial conditions

$$
\left\{\begin{array}{c}
u(M, t) \in U^{[0, T]} \\
\left.\forall t \in[0, T] u\right|_{\partial_{1} \Omega}=u_{d} \\
\left.\forall M \in \Omega u\right|_{t=0}=0
\end{array}\right.
$$

- equilibrium equations

$$
\left\{\begin{array}{l}
\sigma \in S^{[0, T]} \\
\forall t \in[0, T] ; \forall u^{*} \in U_{0} \int_{\Omega} \operatorname{Tr}\left[\sigma \varepsilon\left(u^{*}\right)\right] d \Omega=\int_{\Omega} f_{d}^{T} u^{*} d \Omega+\int_{\partial_{2} \Omega} F_{d}^{T} u^{*} d S
\end{array}\right.
$$

- constitutive relation

$$
\forall t \in[0, T] ;\left.\forall M \in \Omega \sigma\right|_{t}=A\left(\left.\varepsilon(\dot{u})\right|_{\tau} ; \tau \leq t\right)
$$

In that formulation:

- $U^{[0, T]}$ designates the space of the displacement fields and $S^{[0, T]}$ the space of the stress fields. Generally these spaces are chosen in order to impose the condition that the energy of the fields be bounded on $\Omega$ at each time $t \in[0, T] . U_{0}$ is the trial space for the displacement:

$$
U_{0}=\left\{u^{*} \text { such that } u \in \text { Uand }\left.u\right|_{\partial_{1} \Omega}=0\right\}
$$

where $U$ is similar to $U^{[0, T]}$ for the fields which are independent of time;

- $\mathbf{A}$ is an operator which depends on the material and charaterizes the constitutive relation.

\section{Finite element approximation}

The discretization of the problem to be solved is classical. In space, a finite element model is used. For a given $t$, the finite element approximation $u_{h}$ of the displacement field is such that:

$$
u_{h} \in U_{h}
$$

where $U_{h}$ is the discretized space chosen for $U$.

The time interval $[0, T]$ is discretized into $n$ subintervals $\Delta_{i}=\left[t_{i-1}, t_{i}\right]$, $\Delta=\left\{0=t_{0}<t_{1}<\ldots<t_{n}=T\right\}$

Classically, under the assumption that the displacement field is linear over each time increment, the approximate nonlinear problem to be solved at each time step $t_{i}$ is: 
Find $\left(u_{h}^{i}, \sigma_{h}^{i}\right)$ such that:

$$
\begin{gathered}
u_{h}^{i} \in U_{h} \text { and } u_{h}^{i}=u_{d}\left(t_{i}\right) \text { on } \partial_{1} \Omega \\
\int_{\Omega} \operatorname{Tr}\left[\sigma_{h}^{i} \varepsilon\left(u_{h}{ }^{*}\right)\right] d \Omega=\int_{\Omega} f_{d}\left(t_{i}\right)^{T} u_{h}{ }^{*} d \Omega+\int_{\partial_{2} \Omega} F_{d}\left(t_{i}\right)^{T} u_{h}{ }^{*} d S
\end{gathered}
$$

for each $u_{h}^{*} \in U_{h, 0}$

$$
\sigma_{h}^{i}-\sigma_{h}^{i-1}=A_{i}\left(\varepsilon\left(u_{h}^{i}-u_{h}^{i-1}\right)\right)
$$

where $U_{h, 0}=U_{0} \cap U_{h}$ and $A_{i}$ is the discretized operator which charaterizes the constitutive relation.

The non linear problem (4), (5) and (6) is solved using a Newton-Raphson algorithm. Thus, an error related to the precision of the algorithm is introduced. In general, the pair $\left(u_{h}^{i}, \tilde{\sigma}_{h}^{i}\right)$ given by the finite element solvers satisfies (4) and (6) exactly and (5) approximately. It is easy to perform an additional linear resolution to build a stress $\sigma_{h}^{i}$ which satisfies (5) exactly. Under the assumption that the loading is linear over each time increment, the finite element solution is easily completed on $[0, T]$. This solution will be called $\left(u_{h}, \sigma_{h}\right) .\left(u_{h}, \sigma_{h}\right)$ satisfies (4) and (5) exactly and (6) approximately.

\section{Error on the constitutive relation}

The principle of the error on the constitutive relation consists of dividing the equations of the problem to be solved into two groups (Ladevèze and Leguillon, 1983):

- the admissibility conditions: the kinematic constraints and initial conditions (1) and the equilibrium equations (2);

- the constitutive relation (3).

An approximate solution $s_{a d}=\left(u_{K A}, \sigma_{S A}\right)$ for the problem to be solved must satisfy the admissibility conditions ( $u_{K A}$ satisfies (1) and $\sigma_{S A}$ satisfies (2)). The quality of this approximate solution is quantified by the non-verification of the constitutive relation (3).

Drucker's error on the constitutive relation

For models which satisfy Drucker's inequality (Drucker, 1964) strictly, the quality of an admissible pair $s_{a d}=\left(u_{K A}, \sigma_{S A}\right)$ is evaluated by the error measure $e$ (Ladevèze et al., 1986; Gallimard et al., 1996):

$$
e=\sup _{t \in[0, T]}\left[\int_{\Omega} \eta\left(t, s_{a d}\right) d \Omega\right]^{1 / 2}
$$


EC

where

18,7

$$
\eta\left(t, s_{a d}\right)=\int_{0}^{t}\left(\sigma_{S A}-\sigma_{K A}\right)^{T}\left(\dot{\varepsilon}_{S A}-\dot{\varepsilon}_{K A}\right) d t
$$

and

$$
\left\{\begin{array}{c}
\dot{\varepsilon}_{K A \mid t}=\varepsilon\left(\dot{u}_{K A \mid t}\right) \\
\sigma_{K A \mid t}=A\left(\dot{\varepsilon}_{K A \mid \tau} ; \tau \leq t\right) \\
\sigma_{S A \mid t}=A\left(\dot{\varepsilon}_{S A \mid \tau} ; \tau \leq t\right)
\end{array}\right.
$$

Remark: To calculate $\dot{\varepsilon}_{S A \mid t}$, it is necessary to invert the constitutive relation.

We also define the contribution of a time interval $[0, t]$ to the global error:

$$
e_{[0, t]}=\sup _{\tau \in[0, t]} \int_{\Omega} \eta\left(\tau, s_{a d}\right) d \Omega
$$

and a relative error:

$$
\begin{aligned}
\varepsilon & =e / D \\
D^{2} & =2 \int_{0}^{T} \int_{\Omega}\left[\sigma_{K A}^{T} \dot{\varepsilon}_{K A}+\sigma_{S A}^{T} \dot{\varepsilon}_{S A}\right] d \Omega d t
\end{aligned}
$$

\section{Construction of an admissible solution}

The difficulty is to derive an admissible solution $s_{a d}=\left(u_{K A}, \sigma_{S A}\right)$ from the finite element solution $\left(u_{h}, \sigma_{h}\right)$. For the displacement, we simply choose $u_{K A}=u_{h}$. For the stress, we follow the method described in Ladevèze et al. (1986) and Gallimard et al. (1996), which uses the construction techniques developed in elasticity (Ladevèze et al., 1991). We briefly recall the main ideas of this construction.

In a first step, we derive from the finite element solution $\sigma_{h}^{i}(M)$ at $t_{i}$ a stress field $\sigma_{S A}^{i}(M)$ which satisfies the equilibrium equation at $t_{i}$ exactly (2):

$$
\left\{\begin{array}{l}
\sigma_{S A}^{i} \in S \\
\forall u^{*} \in U_{0} \int_{\Omega} \operatorname{Tr}\left[\sigma_{S A}^{i} \varepsilon\left(u^{*}\right)\right] d \Omega=\int_{\Omega} f_{d}\left(t_{i}\right)^{T} u^{*} d \Omega+\int_{\partial_{2} \Omega} f_{d}\left(t_{i}\right)^{T} u^{*} d S
\end{array}\right.
$$

where $S$ is similar to $S^{[0, T]}$ for the fields which are independent of time.

In a second step, we interpolate the balanced fields linearly over each time step. Under the assumption that the loading is linear over each time step (an assumption which, in practice, is not very restrictive), the constructed field is admissible at each instant.

The construction of $\sigma_{S A}^{i}$ is performed in two substeps.

Substep 1. We build on the edges of each element $E$ of the mesh the traction forces $\hat{F}_{h}^{i}$ which represent the stress vectors on the edges of the element.

$$
\sigma_{S A \mid E} n=\eta_{E} \hat{F}_{h}^{i} \text { on } \partial E
$$

(the function $\eta_{E}$ depends on the element $E$; it can assume two constant values: +1 or -1 . Moreover, for two adjacent elements, we prescribe $\eta_{E}+\eta_{E}^{\prime}=0$ on the common edge $\Gamma_{E E^{\prime}}$.) 
Naturally, on the edges belonging to $\partial_{2} \Omega, \eta_{E} \hat{F}_{h}^{i}=F_{d}\left(t_{i}\right)$ is prescribed. Moreover, the tractions $\hat{F}_{h}^{i}$ are constructed such that each element of the mesh is in equilibrium:

$$
\text { for each rigid body displacement } u^{S} \int_{E} f_{d}\left(t_{i}\right)^{T} u^{S} d E+\int_{\partial E} \eta_{E}\left(\hat{F}_{h}^{i}\right)^{T} u^{S} d \Gamma=0 \text {. }
$$

The key to the method is the prolongation condition which links $\sigma_{S A}^{i}$ to the finite element solution:

For each element $E$ in the mesh and for each node $j \int_{E}\left(\sigma_{h}^{i}-\sigma_{S A}^{i}\right)^{T} \varepsilon\left(\phi_{j}\right) d E=0$

where $\phi_{j}$ is the shape function associated with node $j$.

The main lines of the construction of $\hat{F}_{h}^{i}$ are given in the Appendix.

Substep 2. $\sigma_{S A}^{i}$ is built on each element $E$ from the element tractions $\hat{F}_{h}^{i}$ as the solution to the local problem:

$$
\begin{cases}\operatorname{div} \sigma_{S A}^{i}+f_{d}\left(t_{i}\right)=0 & \text { in } E \\ \sigma_{S A}^{i} n=\eta_{E} \hat{F}_{h}^{i} & \text { on } \partial E\end{cases}
$$

Two techniques may be used. The first one, described in Ladevèze et al. (1991), consists of determining a simple polynomial solution. More precisely, each triangular element is subdivided into three subtriangles built on the inertia center and the three vertices. We assume that on each subtriangle $\sigma_{S A}^{i}$ is linear for linear elements and quadratic for quadratic elements. This construction is quasi-explicit and $\sigma_{S A}^{i}$ is a strictly admissible stress field. This technique leads to the standard version of the estimator of the error on the constitutive relation. This error estimator will be designated by $e^{\text {std }}$ (the corresponding relative error is designated by $\varepsilon^{s t d}$ ).

A second technique consists of constructing on $E$ the stress field $\sigma_{S A}^{i}$ as a solution to the following problem:

$$
\left\{\begin{array}{c}
\text { Find } \sigma_{S A}^{i} \text { that satisfies }(17) \\
\text { such that } \sigma_{S A}^{i} \text { minimizes } \int_{E} \operatorname{Tr}\left[\sigma \mathbf{K}^{-1} \sigma\right] d E
\end{array}\right.
$$

where $\mathbf{K}$ is a definite symmetric positive operator.

The dualisation of this problem leads to:

Find $v_{E}^{i}$ a displacement field defined on $E$

for each $v^{*}$ defined on $E$ :

$$
\begin{aligned}
& \int_{E} \operatorname{Tr}\left[\left(\mathbf{K} \varepsilon\left(v_{E}^{i}\right)\right) \varepsilon\left(v^{*}\right)\right] d E=\int_{E} f\left(t_{i}\right)_{d}^{T} v^{*} d E+\int_{\partial E}\left(\hat{F}_{h}^{i}\right)^{T} v^{*} d S \\
& \sigma_{S A \mid E}^{i}=\mathbf{K} \varepsilon\left(v_{E}^{i}\right)
\end{aligned}
$$


This problem is solved numerically on $E$ by the finite element method. We use only one finite element of degree $p+k$ (where $p$ is the degree of the initial finite element analysis and $k$ a positive integer). This technique, which yields an approximate admissible field, was introduced in Strouboulis and Haque (1992) for thermal problems. The corresponding version of the error is called $p+k$ and the estimator is designated by $e^{p+k}$. (The corresponding relative error is designated by $\varepsilon^{p+k}$.)

Remark: a priori this technique works with all definite symmetric positive operator K. In practical computations we use operators linked with the F.E. analysis, for instance the Hooke operator, the tangent rigidity at $t_{i}$ or at $t_{i-1}$. In practice the numerical examples show that the obtained error measure is little sensitive to this choice.

\section{Enhanced Drucker constitutive error estimator}

\section{Principles}

An enhanced version of the Drucker constitutive error estimator, derived from a previous work in elasticity (Ladevèze and Rougeot, 1997), was presented in Gallimard et al. (2000). The aim is to optimize the construction of the admissible field $\sigma_{S A}$ in order to improve the quality of the associated error estimator.

The principle of the method may be described as follows:

- The prolongation condition (15) is weakened by applying it only to the non-vertex nodes:

$$
\begin{aligned}
& \forall E \in T_{h} \text { and for each non-vertex node } j \\
& \int_{E}\left(\sigma_{h}^{i}-\sigma_{S A}^{i}\right)^{T} \varepsilon\left(\phi_{j}\right) d E=0
\end{aligned}
$$

As a result, on each element edge $\Gamma$ the traction $\hat{F}$ can be split into two parts:

$$
\left.\hat{F}\right|_{\Gamma}=\left.\hat{R}\right|_{\Gamma}+\left.\hat{H}\right|_{\Gamma}
$$

$\left.\hat{H}\right|_{\Gamma}$ is a traction with zero resultant force and moment which is completely determined by the weak prolongation condition (19).

$\left.\hat{R}\right|_{\Gamma}$ is a traction which depends on two arbitrary vectors $R_{1 \mid \Gamma}$ and $R_{2 \mid \Gamma}$ which need to satisfy only the global equilibrium (14).

- For each set of $\left.\hat{R}\right|_{\Gamma}$ which satisfies the equilibrium conditions, a stress field $\sigma_{S A}^{i}(\mathbf{R})$ that belongs to $S$ can be constructed using the techniques defined in the preceeding paragraph. $\mathbf{R}$ is the set of vectors $R_{j \mid \Gamma}$ $(j=1,2)$. If we assume that $\sigma_{S A}^{e n h, i-1}$ has already been calculated, $\mathbf{R}_{\text {opt }}$ is determined by minimizing the error contribution on each time step $\left[t_{i-1}, t_{i}\right]$ approximately:

$$
J(\mathbf{R})=\int_{\Omega} \int_{t_{i-1}}^{t_{i}}\left(\sigma_{S A}(\mathbf{R})-\sigma_{K A}\right)^{T}\left(\dot{\varepsilon}_{S A}(\mathbf{R})-\dot{\varepsilon}_{K A}\right) d t d \Omega
$$


where

$$
\sigma_{S A}(\mathbf{R})=\frac{t-t_{i-1}}{t_{i}-t_{i-1}} \sigma_{S A}^{i}(\mathbf{R})+\frac{t_{i}-t}{t_{i}-t_{i-1}} \sigma_{S A}^{e n h, i-1}
$$

The enhanced balanced stress at $t_{i}$ is designated by $\sigma_{S A}^{e n h, i}=\sigma_{S A}^{i}(\mathbf{R})$. The calculated stress field on $[0, T]$ is designated by $\sigma_{S A}^{e n h}$. The details of this technique can be found in Gallimard et al. (2000), together with examples which show that a very good quality error estimator is obtained. In the following sections, this enhanced error estimator will be designated by $e^{\text {enh }}$ (the corresponding relative error will be designated by $\varepsilon^{e n h}$ ).

\section{Variants to the enhanced estimator}

In practice, the optimization is not performed over the whole set of element edges. It is sufficient to perform the minimization on the element edges belonging to "sensitive" zones, e.g. high stress gradient, transition zone between plastic and elastic regions, deformed elements, ... In the other regions, we use the standard densities calculated by the method presented in the Appendix. The choice of the regions where the optimization is performed obviously plays an important role in the method.

Two other parameters may a priori have an influence on the quality of the stress field $\sigma_{S A}$ obtained:

(1) The number of integration points $n_{\Delta}$ used on $\Delta_{i}=\left[t_{i-1}, t_{i}\right]$ to evaluate the function (21).

(2) The criterion chosen to stop the conjugate gradient algorithm used to solve the optimization problem.

The purpose of the following sections is to study the influence of these parameters on the quality of the associated error estimator.

Definition of the numerical tests

The numerical tests presented were performed on the structure shown in Figure 1. This structure is subjected to a prescribed displacement: $\vec{u}_{d}=u_{0} \cdot t \cdot \vec{x}$ (where $u_{0}$ is a scalar quantity).

The material parameters are:

- Young's modulus $E=200 . E 3 \mathrm{Mpa}$,

- Poisson's ratio $\nu=0.3$,

- Initial yield stress $\sigma_{0}=240 \mathrm{Mpa}$,

- Isotropic hardening law $R=500 p^{1 / 2}$.

Two calculations were performed: the first on a quasi-regular mesh (Figure 2 $(2,028 \mathrm{DoF})$ with six time increments), the second on a refined mesh (Figure 3 $(9,672 \mathrm{DoF})$ with 16 time increments). The meshes were made of 6-node triangular elements. The Von Mises stresses at the end of the loading are shown on Figure 4. 
Figure 1 .

Problem to be solved

Figure 2.

Bracket - Calculation 1
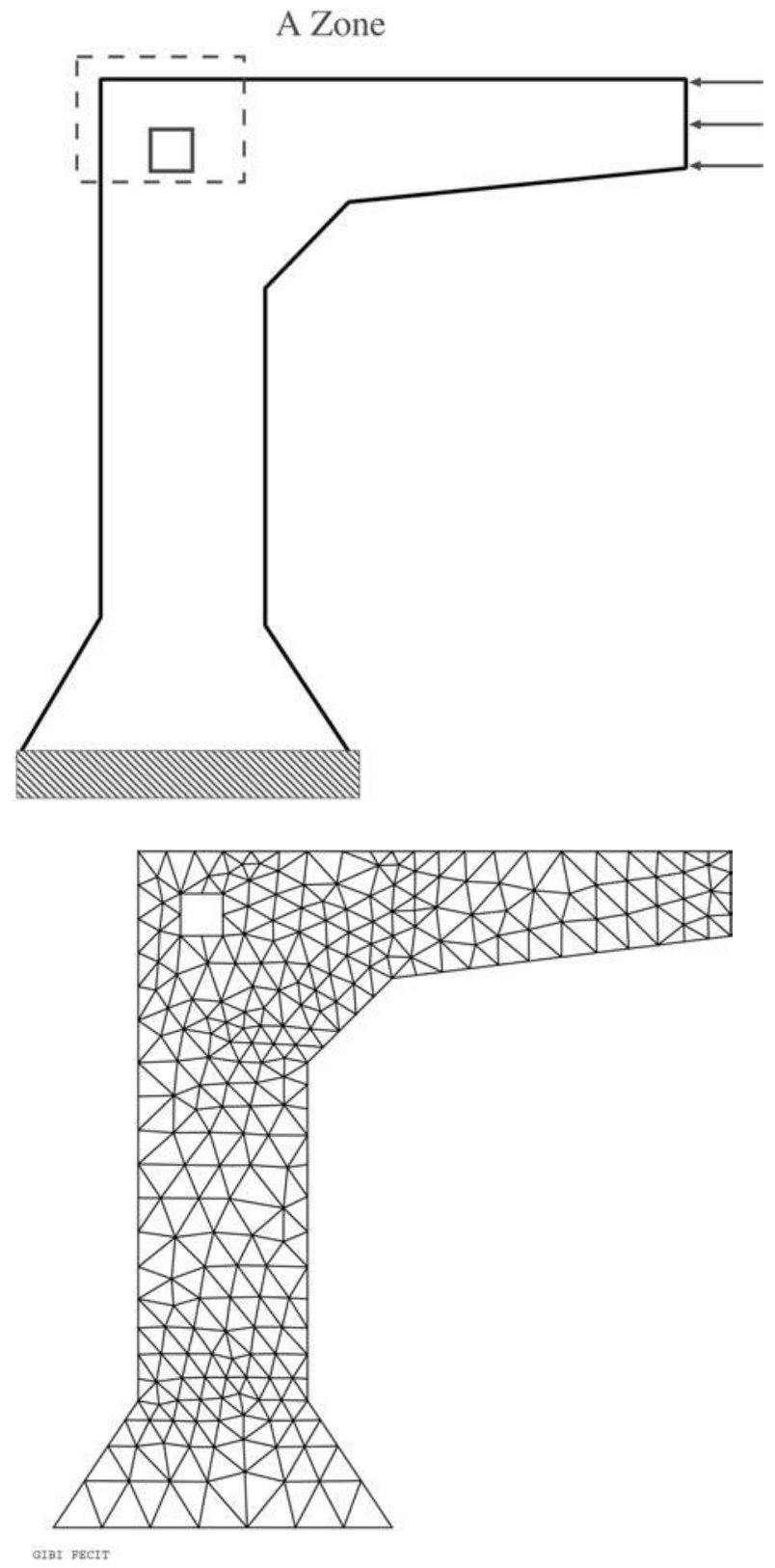

The values of the error estimators $e^{s t d}$ and $e^{p+k}$ (as well as the relative values $\varepsilon^{s t d}$ and $\varepsilon^{p+k}$ ) for Calculations 1 and 2 are summarized in Table I.

Stopping criterion and influence of $n_{\Delta}$

In the examples studied, the function $J(\mathbf{R})$ decreases rapidly during the first iterations of the conjugate gradient technique. Since our objective is to make 


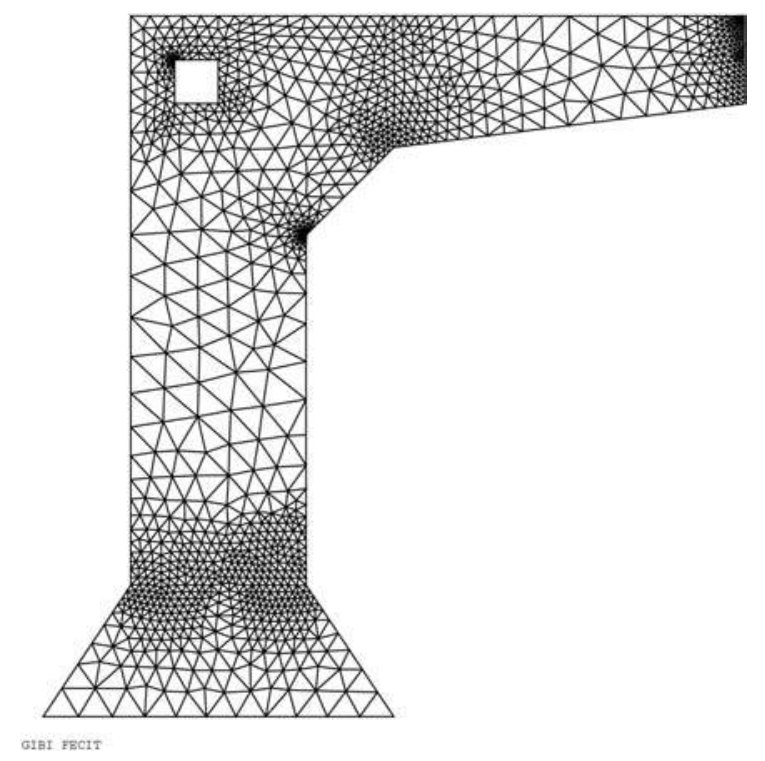

Figure 3.

Bracket - Calculation 2

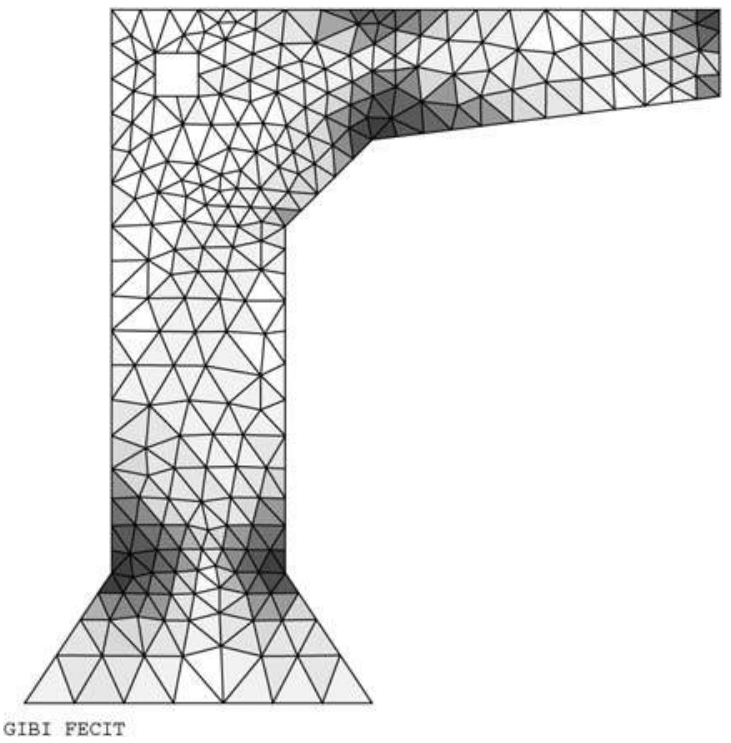

VAL - ISO

$>1.66 \mathrm{E}+01$

$<2.89 \mathrm{E}+02$

60.

$1.20 \mathrm{E}+02$

$1.80 \mathrm{E}+02$

$2.40 \mathrm{E}+02$

$2.44 \mathrm{E}+02$

$2.48 \mathrm{E}+02$

$2.52 \mathrm{E}+02$

$2.56 \mathrm{E}+02$

$2.60 \mathrm{E}+02$

$2.64 \mathrm{E}+02$

$2.68 \mathrm{E}+02$

$2.72 \mathrm{E}+02$

$2.76 \mathrm{E}+02$

2. $80 \mathrm{E}+02$
Figure 4. Bracket - Calculation 1

- Von Mises stress Initial yield stress 240Mpa

$J(\mathbf{R})$ decrease and not to determine the best possible value of $\mathbf{R}$, it is not necessary to iterate until convergence. The stopping criterion of the conjugate gradient technique is based on the mean variation of $J(\mathbf{R})$ during two consecutive iterations.

The study of the influence of the stopping criterion was performed on Calculation 1 (Table II) (The optimization was performed on the whole mesh 
with $n_{\Delta}=8$ ). A stopping criterion of 5 per cent seems sufficient to obtain a great improvement: the error decreased from $e^{p+k}=1.09$ to $e^{e n h}=0.51$. The influence of $n_{\Delta}$ for the calculation is shown in Table III. (The optimization was performed for the whole mesh with a stopping criterion equal to 1.10-4.) We observe that this parameter has very little influence on the error estimator.

Criterion for choosing the patches

Let us define the error density $d_{E}$ on an element $E$ of the mesh $T_{h}$ :

$$
d_{E}=\frac{e_{E}}{\sqrt{m e s(E)}}
$$

where mes $(E)$ is the area of element $E$.

Let $d_{\max }$ be the maximum density:

$$
d_{\max }=\sup _{E \in T_{h}} d_{E}
$$

The simplest criterion to determine the "rough" zones and choose the edges $\Gamma$ where $\hat{R}_{\Gamma}$ must be optimized is to calculate the error $e^{p+k}$ and to determine the elements where the error density is greater than a fraction $\alpha$ of $d_{\max }$. The selected elements with common edges are gathered together into element patches. It is useful to add one or two layers of elements around each patch in order to increase the chance of minimizing the error contribution on elements with a high error density. Then, the patches with common interior edges are gathered together, yielding $n$ patches $\omega_{k}^{\alpha}$. The standard tractions are prescribed on the edge of each patch $\omega_{k}^{\alpha}$; then $J_{\mid} \omega_{k}^{\alpha}$ is minimized.

Table I.

Error - Calculations 1

Calculation

and 2

$e^{s t d}$
$\varepsilon^{s t d}$
$e^{p+k}$
$\varepsilon^{p+k}$

1

1.32

$26.3 \%$

1.09

$23.5 \%$

\section{2}

0.64

$15.0 \%$

0.48

$11.6 \%$
Table II.

Influence of the stopping criterion: Calculation 1

\begin{tabular}{lcccccc}
\hline Stopping criterion & $2.10-1$ & $10-1$ & $5.10-2$ & $10-2$ & $10-3$ & $10-4$ \\
\hline$e^{\text {enh }}$ & 0.71 & 0.61 & 0.51 & 0.44 & 0.40 & 0.39 \\
\hline
\end{tabular}

\begin{tabular}{lcccc}
\hline$n_{\Delta}$ & 1 & 2 & 4 & 8 \\
\hline$e^{\text {enh }}$ & 0.41 & 0.41 & 0.40 & 0.39 \\
$\varepsilon^{\text {enh } \%}$ & 10.2 & 10.1 & 10.0 & 9.8 \\
\hline
\end{tabular}


The default values considered are $\alpha=0$ (all elements are included in the function to be minimized), the stopping criterion of the minimization algorithm is $10^{-2}$ and $n_{\delta}$ is taken equal to 1 .

\section{Influence of the choice of patches}

The results obtained for Calculation 1 with the error estimator $e^{e n h}$ are shown in Table IV. We observe that, on this coarse mesh with a large plastic region, the improvement due to the error estimator $e^{e n h}$ is significant, even for a coefficient $\alpha=0.5\left(e^{p+k}=1.09\right.$ and $\left.e^{e n h}=0.59\right)$. The patches chosen are represented on Figure 5).

On Calculation 2, which was performed on a refined mesh, we observe that for a coefficient $\alpha=0.5$ we again obtain a significant improvement in the error estimation (Table V). The patches used for $\alpha=0.5$ are shown on Figure 6 .

\section{Adaptive control technique}

The adaptive technique proposed relies on an initial coarse calculation. From this first calculation, we determine the parameters (mesh, time discretization) of a second calculation in order to obtain a given precision $\varepsilon_{0}$ for as little cost as possible. The error measure $\varepsilon$ is global in space and time and takes into account

\begin{tabular}{|c|c|c|c|c|c|c|c|}
\hline Coef. $\alpha$ & 0.5 & 0.4 & 0.3 & 0.2 & 0.1 & 0.0 & \\
\hline $\begin{array}{l}e^{e n h} \\
\left(e^{p+k}-e^{e n h}\right) / e^{p+k} \\
\left(e^{s t d}-e^{e n h}\right) / e^{s t d}\end{array}$ & $\begin{array}{l}0.59 \\
46 \% \\
55 \%\end{array}$ & $\begin{array}{l}0.55 \\
49 \% \\
58 \%\end{array}$ & $\begin{array}{l}0.48 \\
56 \% \\
64 \%\end{array}$ & $\begin{array}{l}0.48 \\
56 \% \\
64 \%\end{array}$ & $\begin{array}{l}0.44 \\
60 \% \\
67 \%\end{array}$ & $\begin{array}{l}0.44 \\
60 \% \\
67 \%\end{array}$ & $\begin{array}{r}\text { Table IV. } \\
\text { Influence of } \\
\alpha-\text { Calculation } 1\end{array}$ \\
\hline
\end{tabular}

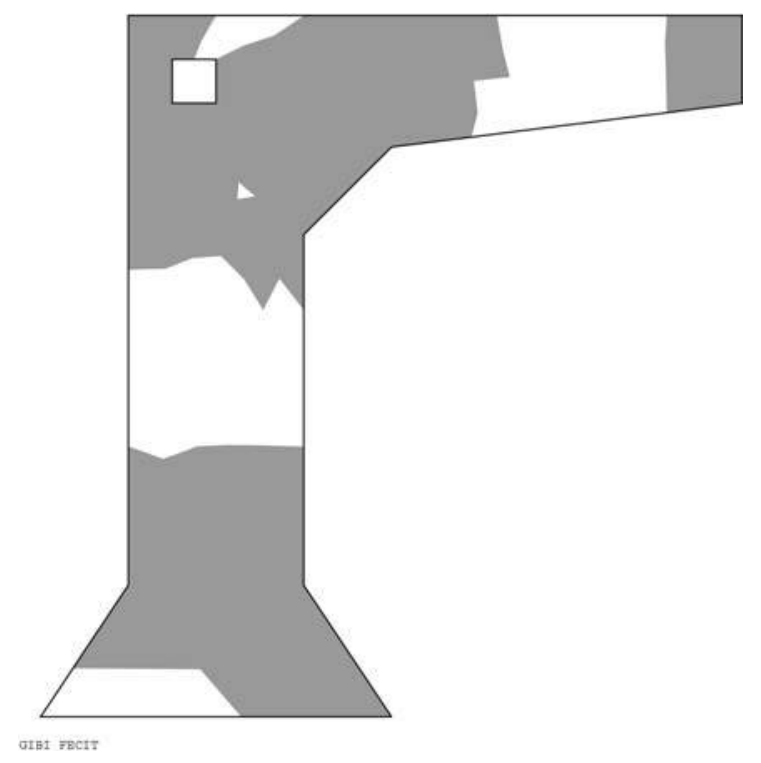

Figure 5. Calculation 1 
the mesh quality, the quality of the time discretization and the precision of the stopping criterion of the iterative technique used. This global information is not sufficient to determine the calculation parameters to obtain the required precision. To determine these parameters, we use tools which we call error indicators which allow us to evaluate the errors due to the mesh and the errors due to the time discretization.

\section{Error indicators}

We use the time error indicator developed in Gallimard et al. (1996), which is common to the three global error estimators. The calculation of the space error indicator depends on the construction technique of the admissible stress field. We use the definition of the space error indicator given in Ladevèze and Moës (1999). Basically, this indicator is constructed by choosing as the reference problem the problem which is discrete in time and continuous in space. The error occurring between this new reference problem and the finite element solution is due only to the space discretization.

An admissible solution for this problem is the set of pairs $s_{a d}^{i}=\left(u_{K A}^{i}, \sigma_{S A}^{i}\right)$ for $i \in\{1, \ldots, n\}$.

Let us define:

$$
\eta_{\text {space }}\left(s_{a d}^{i}\right)=\left(\sigma_{S A}^{i} 1-\sigma_{K A}^{i+1}\right)^{T}\left(\Delta \varepsilon_{S A}^{i+1}-\Delta \varepsilon_{K A}^{i+1}\right)
$$

Table V.

Influence of $\alpha$ on Calculation 2
Coef. $\alpha$

$e^{e n h}$
$\left(e^{p+k}-e^{e n h}\right) / e^{p+k}$
$\left(e^{s t d}-e^{e n h}\right) / e^{s t d}$

0.5

\subsection{7}

$57.2 \%$

$58.4 \%$

0.4

0.255

$59.1 \%$

$60.3 \%$

0.3

0.192

$69.2 \%$

$70.1 \%$

0.2

0.2

0.167

$73.2 \%$

$74.0 \%$
0.1

0.160

$74.3 \%$

$75.1 \%$

Figure 6.

Calculation $2-5$ patches

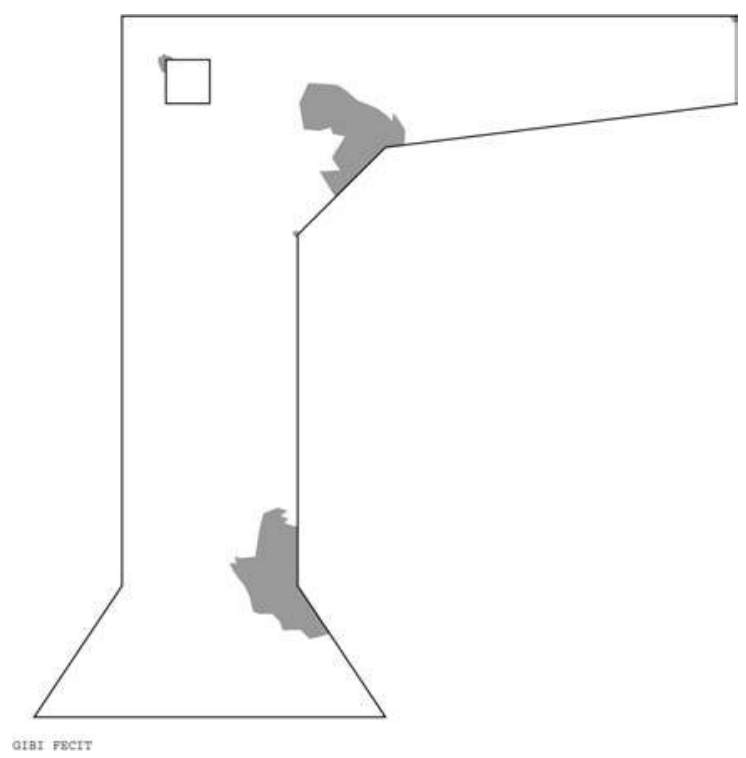


where

and

$$
\Delta x^{i+1}=x_{i+1}-x_{i}
$$

$$
\sigma^{i+1}-\sigma^{i}=A_{i}\left(\varepsilon^{i+1}-\varepsilon^{i}\right)
$$

$\mathbf{A}_{i}$ is the discretized operator of the constitutive relation obtained by an implicit Euler integration scheme.

A new space error indicator may be defined with the enhanced admissible pairs $s_{a d}^{e n h, i}=\left(u_{K A}^{i}, \sigma_{S A}^{e n h, i}\right)$ for $i \in\{1, \ldots, n\}$ :

$$
\left(I_{\text {space }}^{\text {enh }}\right)^{2}=\sup _{k \in\{0, \ldots, n-1\}} \sum_{i=0}^{k}\left(\int_{\Omega} \eta_{\text {space }}\left(s_{a d}^{\text {enh }, i+1}\right) d \Omega\right.
$$

It is possible, like for the global error, to define a relative space error indicator: $i_{\text {space }}^{\text {enh }}$. The space error indicators $i_{\text {space }}^{\text {enh }}$ and $i_{\text {space }}^{\text {enh }}$ are calculated in the same way.

Relation between $\varepsilon, i_{\text {space }}$ and $i_{\text {time }}$

Let us consider the quantity $i_{\text {tot }}$ defined by:

$$
i_{\text {tot }}^{2}=i_{\text {space }}^{2}+i_{\text {time }}^{2}
$$

In Ladevèze and Moës (1998), it is shown numerically that:

$$
i_{\text {tot }}^{s t d}=\sqrt{\left(i_{\text {space }}^{\text {std }}\right)^{2}+i_{\text {time }}^{2}} \simeq \varepsilon^{\text {std }} .
$$

\begin{tabular}{|c|c|c|c|c|c|c|}
\hline & mesh 1 & mesh 2 & mesh 3 & mesh 4 & mesh 5 & \multirow{6}{*}{$\begin{array}{l}\text { Table VI. } \\
\text { Error indicators as a } \\
\text { function of the mesh }\end{array}$} \\
\hline elements & 160 & 220 & 567 & 1,692 & 4,312 & \\
\hline$\varepsilon^{e n h}(\%)$ & 22.02 & 14.53 & 9.95 & 5.21 & 4.68 & \\
\hline ispace $(\%)$ & 22.41 & 14.29 & 8.92 & 3.06 & 1.42 & \\
\hline$i_{\text {time }}(\%)$ & 3.96 & 4.38 & 4.57 & 4.39 & 4.53 & \\
\hline$i_{t o t}^{e n h}(\%)$ & 22.76 & 14.94 & 10.01 & 5.35 & 4.75 & \\
\hline Time steps & 1 & \multicolumn{2}{|c|}{2} & 4 & 8 & \multirow{5}{*}{$\begin{array}{r}\text { Table VII. } \\
\text { Error indicators as a } \\
\text { function of the time } \\
\text { discretization }\end{array}$} \\
\hline$\varepsilon^{e n h} \%$ & 19.55 & & & 14.39 & 14.55 & \\
\hline$i_{\text {space }}^{\text {enh }} \%$ & 12.44 & & & 14.30 & 14.80 & \\
\hline$i_{\text {time }} \%$ & 17.94 & & & 2.91 & 1.29 & \\
\hline$i_{t o t}^{e n h} \%$ & 21.83 & & & 14.59 & 14.86 & \\
\hline
\end{tabular}

The same property is observed for the "enhanced" and " $\mathrm{p}+\mathrm{k}$ " quantities. On Table VI, we increase the quality of the mesh for a given time discretization. On Table VII, we increase the number of time steps for a given mesh. 


\section{Adaptation of the calculation parameters}

In the following section, since the adaptive technique proposed is the same for the three error estimators, we will omit the exponents $s t d, p+k$ and $e n h$.

For a prescribed global error $\varepsilon_{0}$, the following procedure may be defined:

- An initial analysis over the time interval $[0, T]$ is performed on a coarse mesh $T_{h}$ for a time discretization $\Delta$. Then, the global error $\varepsilon$, the time indicator $i_{\text {time }}$ and the space indicator $i_{\text {space }}$ are calculated.

- A new mesh $T_{h}^{*}$ and a new time discretization $\Delta^{*}$ are determined in order to minimize a cost function $f\left(N^{*}, M^{*}\right)=\left(M^{*}\right)^{\beta} \cdot\left(N^{*}\right)^{\gamma}$ with the restriction:

$$
\left(i_{\text {space }}^{*}\right)^{2}+\left(i_{\text {time }}^{*}\right)^{2}=\varepsilon_{0}^{2}
$$

where $N^{*}$ is the number of elements in $T_{h}^{*}$ and $M^{*}$ is the number of time steps in $\Delta^{*}$.

This minimization problem is solved by using techniques introduced in Ladevèze et al. (1991) and later in Coorevits et al. (1994) and Ladevèze and Moës (1999).

Remarks:

- For the 6-node triangular elements that we use in the examples, the value of $\beta$ is set to 1 and the value of $\gamma$ is set to 2 .

- If the prescribed global error $\varepsilon_{0}$ is too small compared to the global error $\varepsilon$ obtained in the initial calculation, it is, in practice, difficult to determine the parameters of the optimized calculation directly. One or more intermediate calculations must be performed with target errors equal to $\theta \varepsilon$ (where, for instance, $\theta=0.25$ ).

\section{Examples}

To illustrate this procedure, we reexamine the example of the structure subjected to a prescribed horizontal displacement 1 . The initial analysis (Figure 7) is performed with two time steps (this calculation is referred to as Calculation 0).

The three error estimators presented in the previous section are used in succession with the adaptive procedure. The parameters chosen for the calculation of $\varepsilon^{e n h}$ are: $n_{\Delta}=1$, stopping criterion $=5.10-2$ and $\alpha=0.5$.

The prescribed global error $\varepsilon_{0}$ is 5 per cent.

In Table VIII, we show the error, the number of nodes and the number of time steps obtained for the optimized calculation. Figures 8-10 show the resulting discretizations.

A zoom is performed on Zone A to study the mesh more precisely. The error measure $\varepsilon^{\text {std }}$ is very large in the presence of a high stress gradient and that leads to an overrefinement of Zone A (Figure 11). Moreover, the high error gradient in this zone leads to the creation by the mesh generator of deformed 


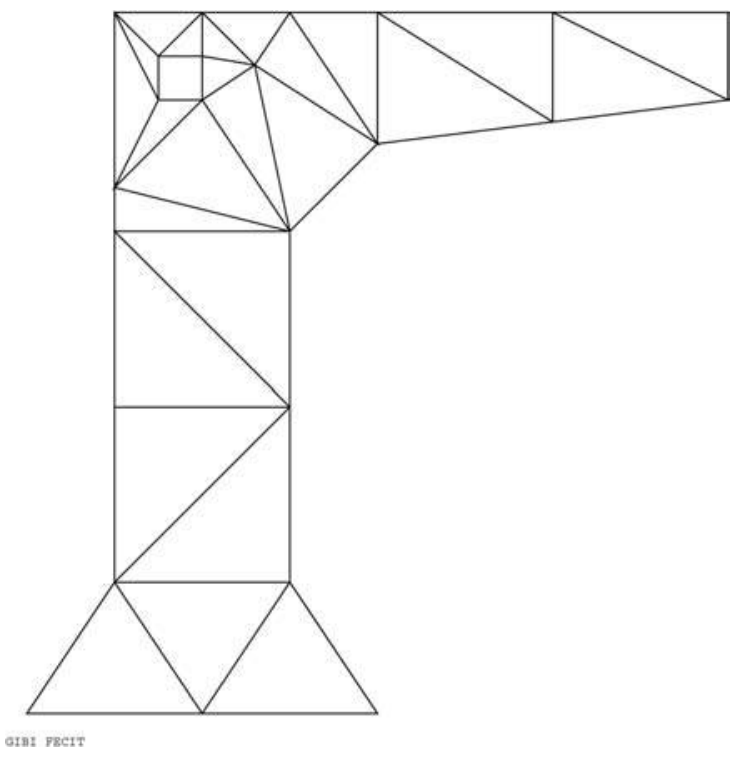

Figure 7.

Calculation 0

\begin{tabular}{lccc}
\hline & $e^{\text {std }}$ & $e^{p+k}$ & $e^{\text {enh }}$ \\
\hline Error (\%) & 4.89 & 5.01 & 4.70 \\
NDDL & 7,714 & 6,356 & 5,206 \\
Number of steps & 7 & 7 & 7
\end{tabular}

Table VIII.

Optimized calculation
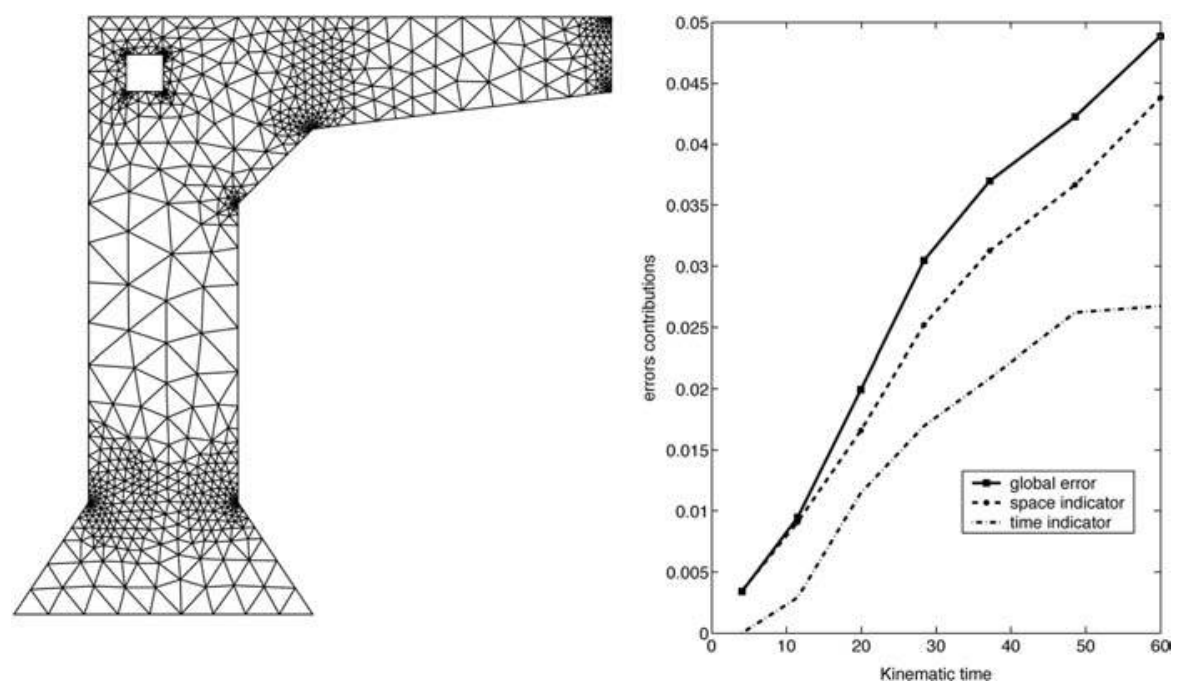

Figure 8.

$e^{\text {std }}-$ optimized calculation 
Figure 9.

$e^{p+k}-$ optimized calculation

Figure 10.

$e^{e n h}-$ optimized calculation

Figure 11.

Optimized calculation $e^{\text {std }}$ - mesh zone A
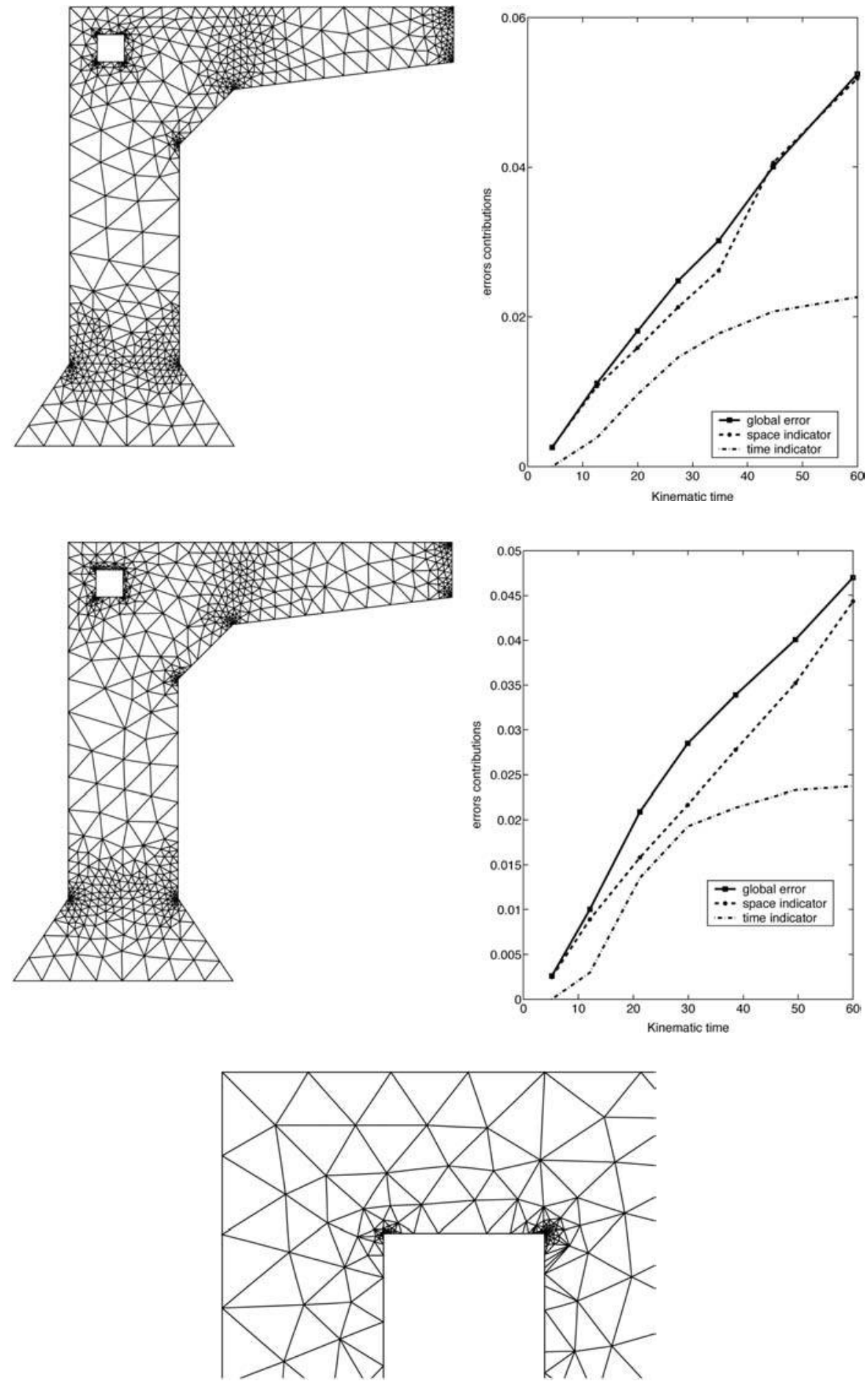
elements. For the error estimator $\varepsilon^{p+k}$, the overrefinement is, again, important (Figure 12). For the error $\varepsilon^{e n h}$ (Figure 13), the minimization performed in the zones where the error is overestimated enables this phenomenon to be corrected, resulting in a more balanced mesh. Thus, the size variations of the elements are more easily dealt with by the mesh generator.

The evolutions of some quantities of interest were also studied during the evolution of the adaptive procedure. Calculation 1 corresponds to the discretization obtained after one iteration of the adaptive technique. Calculation 2 corresponds to the discretization obtained when the global error reaches approximately 10 per cent.

In Table IX, we represented the evolution of the plastic work calculated from the finite element solution. The three calculations converge toward the same result.

In Table $\mathrm{X}$ we represented the evolution of the plastic work calculated from the statically admissible solution. The three calculations converge toward an identical result. But we observe that for calculations 0 and 1, which are very

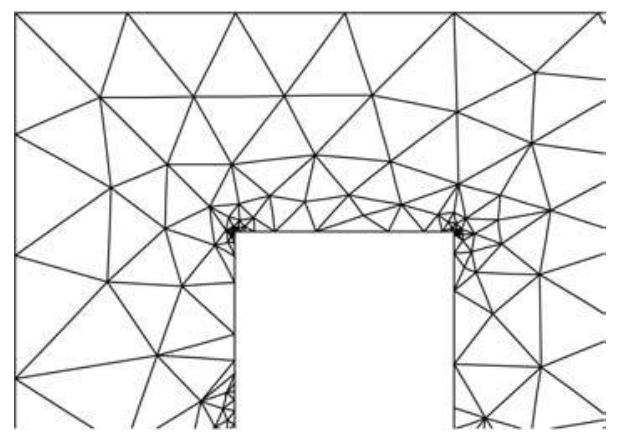

Figure 12. Optimized calculation $e^{p+k}-$ mesh zone A

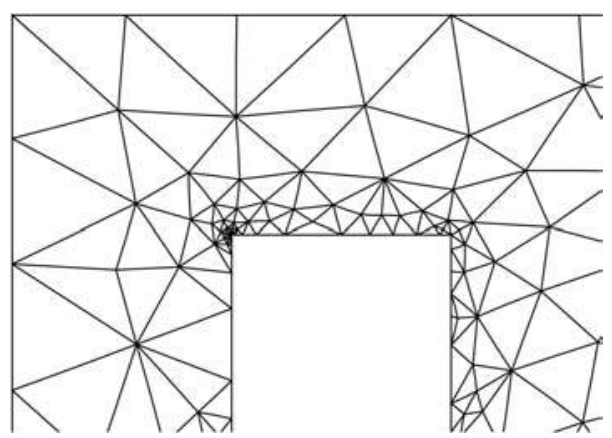

Figure 13.

Optimized calculation $e^{e n h}-$ mesh zone A

\begin{tabular}{lccc}
\hline Calculation & $e^{\text {std }}$ & $e^{p+k}$ & $e^{\text {enh }}$ \\
\hline 0 & 2.66 & 2.66 & 2.66 \\
1 & 2.51 & 2.49 & 2.51 \\
2 & 2.34 & 2.34 & 2.34 \\
3 & 2.33 & 2.33 & 2.33
\end{tabular}

Table IX.

Plastic work during the loading (KA solution) 
coarse, the admissible solution is not good and the improvement due to the optimization technique is very significant.

In Table XI, we show the evolution of the size of the plastic zone (calculated from the finite element solution). Once again, the three calculations converge toward similar values and the values calculated during the initial analysis are very inaccurate.

\section{Conclusion}

In this paper, we have proposed a study of the enhanced error $\varepsilon^{\text {enh }}$ on the constitutive relation in plasticity and shown that the calculation of this error estimator in order to estimate the global quality of the calculation may be restricted to the zones which create difficulties. We have shown that the use of this error estimator and associated error indicators in an adaptive procedure allows us to end up with less refined and more regular meshes while preserving the precision and the quality of the calculation.

Table X. $\quad 1$

\begin{tabular}{lccc}
\hline Calculation & $e^{\text {std }}$ & $e^{p+k}$ & $e^{\text {enh }}$ \\
\hline 0 & 771.5 & 368.0 & 202.0 \\
1 & 46.1 & 23.0 & 12.7 \\
2 & 2.87 & 2.89 & 2.78 \\
3 & 2.45 & 2.45 & 2.46
\end{tabular}

loading (SA solution)

\begin{tabular}{lccc}
\hline Calculation & $e^{\text {std }}$ & $e^{p+k}$ & $e^{\text {enh }}$ \\
\hline 0 & 38.40 & 38.40 & 38.40 \\
1 & 22.09 & 20.95 & 22.20 \\
2 & 19.09 & 19.51 & 19.14 \\
3 & 18.90 & 18.81 & 18.84
\end{tabular}

\section{References}

Babuška, I. and Rheinboldt, W. (1978), "A posteriori estimates for the finite element method”, Int. L. for Num. Meth. in Engrg., Vol. 12, pp. 1597-615.

Babuška, I. and Rheinboldt, W. (1982), "Computational error estimates and adaptive processes for some nonlinear structural problems”, Comp. Meth. in Applied Mech. and Engrg., Vol. 34, pp. 895-937.

Coorevits, P., Dumeau, J. and Pelle, J. (1999), "Control of analyses with isoparametric elements in 2D and 3D”, Int. I. for Num. Meth. in Engrg., Vol. 46, pp. 157-76.

Coorevits, P., Ladevèze, P. and Pelle, J. (1994), "Mesh optimization for problems with steep gradients", Engrg. Computation, Vol. 11, pp. 129-44.

Coupez, T., Fourment, L. and Chenot, J. (1998), "Adaptive solutions in industrial forming process simulation", in Ladevèze. P. and Oden. I. (Eds). Advances in Adabtive Computational Methods in Mechanics. Studies in Applied Mechanics, Vol. 47, Elsevier, London, pp. 349-64.

Drucker, D. (1964), "On the postulate of stability of material in the mechanics of continua", Journal de Mècanique, Vol. 3 No. 2, pp. 235-49. 
Gallimard, L., Ladevèze, P. and Pelle, J. (1996), "Error estimation and adaptivity in elastoplasticity", Int.I. for Num. Meth. in Engrg., Vol. 39, pp. 189-217.

Gallimard, L., Ladevèze, P. and Pelle, J. (1997), "Error estimation and time-space parameters optimization for FEM non-linear computation”, Computers and Structures, Vol. 64, pp. 145-56.

Gallimard, L., Ladevèze, P. and Pelle, J. (2000), "An enhanced error estimator on constitutive relation for plasticity problems”, Computers and Structures, Vol. 78, pp. 801-10.

Huerta, A., Diez, P., Rodriguez-Ferran, A. and Pijaudier-Cabot, G. (1998), "Error estimation and adaptative finite element analysis of softening solids", in Ladevèze. P. and Oden I. (Eds). Advances in Adaptive Computational Methods in Mechanics, Studies in Applied Mechanics, Vol. 47, Elsevier, London, pp. 333-48.

Johnson, C. and Hansbo, P. (1992), "Adaptative finite element methods in computational mechanics", Comp. Meth. in Applied Mech. and Engrg., Vol. 101, pp. 143-81.

Ladevèze, P. and Leguillon D. (1983), "Error estimate procedure in the finite element method and application”, SIAM I. Num. Anal., Vol. 20 No. 3, pp. 485-509.

Ladevèze, P. and Moës, N. (1998), "A posteriori constitutive relation error estimators for nonlinear finite element analysis and adaptive control", in Ladevèze, P. and Oden. I. (Eds). Advances in Adaptive Computational Methods in Mechanics, Studies in Applied Mechanics, Vol. 47, Elsevier, London, pp. 231-56.

Ladevèze, P. and Moës, N. (1999), "Adaptive control for finite element analysis in plasticity", Computers and Structures, Vol. 73, pp. 45-60.

Ladevèze, P. and Pelle, J. (1983), "Méthode de calcul par encadrement des fréquences propres de structures élastiques”, Comptes Rendus Acad. Sci. Paris, Série II, Vol. 296, pp. 1757-60.

Ladevèze, P. and Rougeot, P. (1997), "New advances on a posteriori error on constitutive relation in f.e. analysis”, Comp. Meth. in Applied Mech. and Engrg., Vol. 150, pp. 239-49.

Ladevèze, P., Coffignal, G. and Pelle, J. (1986), "Accuracy of elastoplastic and dynamic analysis", in Babuška, I., Gago, J., de A. Oliveira, E. and Zienkiewicz O. (Eds), Accuracy Estimates and Adaptive Refinements in Finite Element Computations, John Wiley \& Sons, New York, NY, pp. 181-203.

Ladevèze, P., Pelle, J. and Rougeot, P. (1991), "Error estimation and mesh optimization for classical finite elements”, Engrg. Computation, Vol. 8, pp. 69-80.

Ladevèze, P., Rougeot, P., Blanchard, P. and Moreau, J. (1999), "Local error estimators for finite element analysis”, Comp. Meth. in Applied Mech. and Engrg., Vol. 176, pp. 231-46.

Rannacher, R. and Stuttmeier, F. (1999), "A a posteriori error estimation and mesh adaptation for finite element models in elasto-plasticity", Comp. Meth. in Applied Mech. and Engrg., Vol. 176, pp. 331-61.

Strouboulis, T. and Haque, K. (1992), "Recent experiences with error estimation and adaptivity, Part 1: Review of error estimators for scalar elliptic problems", Comp. Meth. in Applied Mech. and Engrg., Vol. 97, pp. 399-436.

Zienkiewicz, O. and Zhu, J. (1987), "A simple error estimator and adaptive procedure for practical enineering analysis”, Int. I. for Num. Meth. in Engrg., Vol. 24, pp. 337-57.

\section{Appendix. Standard recovery method}

The construction of the balanced stress field is performed at each time step. For the sake of simplicity, we will omit the exponent $s t d, i$.

\section{Prolongation condition}

The admissible stress $\sigma_{S A}$ is linked to the finite element stress field by the "strong" prolongation condition defined by the relation (15). This can be written using the equilibrium of $\sigma_{S A}$ : 


$$
\int_{\partial E}\left(\sigma_{S A} \cdot n\right)^{T} \phi_{j} d \Gamma=\int_{E} \sigma_{h}^{T} \varepsilon\left(\phi_{j}\right)-\int_{E} f_{d}^{T} \phi_{j} d E=Q_{E}(j)
$$

This relation introduces the element tractions on the element boundaries. The element traction on element $E$ is designated by $\eta_{E} \hat{F}_{h}$.

- $\hat{F}_{h}$ is defined on the element edges.

- $\eta_{E}$ is a scalar function defined on $E$ such that $\eta_{E}= \pm 1$ and $\eta_{E}+\eta_{E}^{\prime}=0$ on the common edge $\Gamma_{E E^{\prime}}$ of two adjacent elements $E$ and $E^{\prime}$.

With the expression:

$$
\eta_{E} \hat{F}_{h}=\sigma_{S A} \cdot \text { on } \partial E
$$

Equation (30) becomes:

$$
\int_{\partial E} \eta_{E} \hat{F}_{h}^{T} \phi_{j} d \Gamma=\int_{E} \sigma_{h}^{T} \varepsilon\left(\phi_{j}\right)-\int_{E} f_{d}^{T} \phi_{j} d E=Q_{E}(j)
$$

Remark: On an edge $\Gamma \in \partial_{2} \Omega$ we impose $\eta_{E} \hat{F}_{h}=F_{d}$

In Ladevèze and Pelle (1983), it is shown that if $\eta_{E} \hat{F}_{h}$ satisfies the prescribed loading on $\partial_{2} \Omega$ and Equation (32), then the element tractions $\hat{F}_{h}$ are balanced.

Standard construction of $\hat{F}_{h}$

The complete construction can be found in Ladevèze et al. (1991). We will consider only the case where the nodes are internal to $\Omega$.

Non-vertex node: $j \in \Gamma_{E E^{\prime}}$ common edge to $E$ and $E^{\prime}$.

The prolongation condition (32) leads to:

$$
\begin{gathered}
\int_{\Gamma_{E E^{\prime}}} \eta_{E} \hat{F}_{h}^{T} \phi_{j} d \Gamma=Q_{E}(j) \\
\int_{\Gamma_{E E^{\prime}}} \eta_{E}^{\prime} \hat{F}_{h}^{T} \phi_{j} d \Gamma=Q_{E}^{\prime}(j)
\end{gathered}
$$

Writing the finite element equilibrium leads to:

$$
Q_{E}(j)+Q_{E}^{\prime}(j)=0
$$

Therefore,

$$
\int_{\Gamma_{E E^{\prime}}} \hat{F}_{h}^{T} \phi_{j} d \Gamma=\eta_{E}^{\prime} Q_{E}^{\prime}(j)=\eta_{E} Q_{E}(j)
$$

Vertex node $j$ : We write (cf Figure A1):

Figure A1.

Vertex nodes

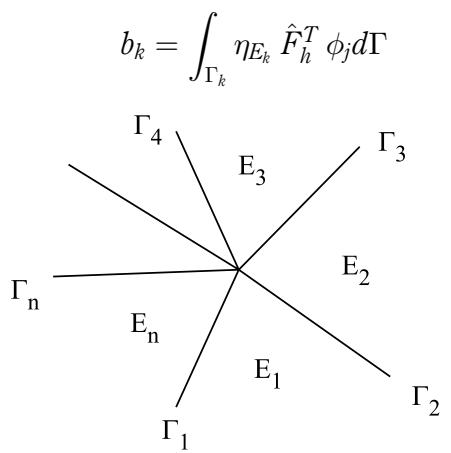


Equation (32) for the elements connected to node $j$ leads to the following linear system:

$$
\left\{\begin{array}{l}
b_{1}-b_{2}=Q_{E_{1}}(j) \\
b_{2}-b_{3}=Q_{E_{2}}(j) \\
b_{3}-b_{4}=Q_{E_{3}}(j) \\
b_{n}-b_{1}=Q_{E_{n}}(j)
\end{array}\right.
$$

This system has an infinity of solutions if $\sum_{k=1}^{k=n} Q_{E_{k}}(j)=0$, a property which occurs if $\sigma_{h}$ satisfies the finite element equilibrium. In practice, $b_{i}$ are chosen in order to minimize:

$$
f\left(b_{1}, b_{2}, \ldots, b_{n}\right) \longrightarrow \sum_{k=1}^{k=n} \frac{\left(b_{k}-b_{k}^{m, h}\right)^{2}}{L_{k}^{2}}
$$

where $L_{k}=\operatorname{mes}\left(\Gamma_{k}\right)$ and $b_{k}^{m, h}=\frac{1}{2} \int_{\Gamma_{b}}\left(\sigma_{h \mid E_{k}} \cdot n_{k}-\sigma_{h \mid E_{k-1}} \cdot n_{k-1}\right) \omega_{j} d \Gamma$.

The element tractions are then recovered by assuming that $\hat{F}_{h}$ is a linear combination of the shape functions on the element edges:

$$
\hat{F}_{h}^{i}=A_{\alpha}^{i} \phi_{\alpha}+A_{\beta}^{i} \phi_{\beta}+\sum_{j \in \bar{I}} A_{j}^{i} \phi_{j}
$$

